\title{
Evaluation of Surgical Treatment in Patients with Total Anomalous Pulmonary Venous Connection: Fifteen Years of Single Institute Experience
}

\author{
Gökmen Akkaya, Çağatay Bilen, Osman Nuri Tuncer, Mehmet Fatih Ayık, Yüksel Atay
}

Ege University Faculty of Medicine, Department of Cardiovascular Surgery, İzmir, Turkey

\begin{abstract}
Background: In this study, we present our fifteen years of outcomes following surgical repair total anomalous pulmonary venous connection (TAPVC). Thereby we sought to assess possible risk factors effects both hospital and late term follow up morbidity and mortality.

Methods: A retrospective study was carried out in the Department of Cardiovascular Surgery of the Ege University Hospital between 2002-2017 by including all patients who were operated on with the diagnosis of TAPVC. Patient's demographic data, preoperative medical data, operative findings and post-discharge findings, echocardiography and computed tomography results were collected.
\end{abstract}

Results: The mean age of the patients was determined as
$9 \pm 11.68$ months. The mean weights of the patients before the operation were found to be $3720 \pm 2042$ gr. Nineteen of the patients were supracardiac, 13 were intracardiac, 8 were infracardiac, and one was mixed type TAPVC. Mean cardiopulmonary by-pass time was $84.78 \pm 25.84$ minutes and mean aortic cross-clamp time was $57.24 \pm 15.17$ minute.

Conclusion: Thanks to developed surgical techniques and medical care, nowadays, surgical treatment may be utilized with similar mortality and morbidity rates regardless of coexisting cardiac defects to all types of TAPVC at every age and provides satisfactory results in late term follow-up.

Keywords: Total anomalous pulmonary venous connection, congenital heart disease, complication

Akkaya G., Bilen Ç., Tuncer ON., Ayık MF., Atay Y. Evaluation of Surgical Treatment in Patients with Total Anomalous Pulmonary Venous Connection: Fifteen Years of Single Institute Experience. EJCM 2018; 06 (4): 128-133. DOI: 10.32596/ejcm.00043

\footnotetext{
Address for Correspondence: Gökmen Akkaya, Ege University Faculty of Medicine, Department of Cardiovascular Surgery, İzmir, Turkey e-mail: akkayagokmen@gmail.com 


\section{Introduction}

Total anomalous pulmonary venous connection (TAPVC) is a distinctly rare disorder and constitutes only $1-3 \%$ among all congenital heart diseases. ${ }^{(1)}$ According to level of connection site, it is classified into three main groups: supracardiac, cardiac, infracardiac; or a mixture of these two.

Basically, TAPVC involves various congenital anomalies which have no connection between any pulmonary vein and the left atrium, contrary to usual anatomical development. Embryologically, TAPVC occurs following a defect while common pulmonary vein incorporating into the back wall of the left atrium and maintaining its connection with the cardinal and umbilicovitelline veins. In such cases, all four pulmonary veins connect to the right atrium directly or via a vertical vein. ${ }^{(2)}$ Therefore, right atrium receives the entire pulmonary venous return additively to the systemic venous return. As a result, left atrium receives no blood flow, therefore survival in postnatal life depends on a shunt which provides blood flow towards left side of the heart between atriums by patent foramen ovale or an atrial septal defect.

However, when left untreated, mortality rate in first year may lead up to $\% 78 .^{(3)}$ Nonetheless, since the initial successful attempt was reported by Muller et al. in 1951, by the help of developing technologies, advanced surgical techniques as well as improved knowledge in hospital care substantially reduced the complications. ${ }^{(4)}$ However, the surgical repair of TAPVC still remains a challenge thereby reported mortality rates ranging between 10$20 \%$ in presence of coexisting cardiac anomalies such as neonatal surgical repair, preoperative pulmonary venous obstruction, mixed anatomic variation, single ventricle physiology, and heterotaxy which are previously indicated as risk factors for poorer outcomes. ${ }^{(5,6)}$ Furthermore, TAPVC is associated with lower birth weight, younger gestational age, and intrauterine growth retardation. ${ }^{(7)}$ In this study, we present our fifteen years of single institute results, thus we aim to assess the risk factors which are worsening the early and late term results in patients with TAPVC.

\section{Materials and Methods}

A retrospective study was carried out in the Department of Cardiovascular Surgery of the Ege University Hospital between 2002-2017 by including all patients who were operated on with the diagnosis of (TAPVC).

The medical data of all patients was collected using the hospital database. A study was undertaken regardless of associated cardiac pathologies of the patients and a total of forty one patients were included in the study.

Patients' demographic data, preoperative medical data, operative findings (operative technique, cardiopulmonary bypass, cross clamp time), hospitalization (duration of intubation, intensive care and hospital stay, usage of nitric oxide, need for extracorporeal membrane oxygenator (ECMO), if the patient is released as open sternum from the operative time until the sternum is closed) and postdischarge findings, echocardiography and computed tomography results were collected.

Patients were divided into four classes using Craig and Darling classification. These are; type 1 supracardiac, type 2 intracardiac, type 3 infracardiac, and type 4 mixed type.

Pulmonary hypertension was classified as grade 0 if the systolic pulmonary pressure was normal, grade 1 if it ranged between $30 \% 50 \%$, grade 2 if it ranged between $50 \% 75 \%$, and grade 3 if it was higher than $75 \%$ of the systolic blood pressure.

Early operative mortality was defined for deaths during the operation, during hospitalization, and within the first 30 days postoperatively. Complications were defined as those requiring medical treatment and requiring surgical intervention. These are identified such as neurological, respiratory, renal, infection, bleeding, arrhythmia, heart failure and thromboembolic events.

All patients underwent surgery via standard cardiopulmonary by-pass. The surgery was performed according to physiopathology under cold cardioplegia. In supra and infracardiac typed TAPVD patients, pulmonary venous sac was opened and was anastomosed side to side with the morphological left atrium. For cardiac TAPVC; 
coronary sinus was made unroofed and the atrial septum was repaired as the pulmonary veins remained in the left atrium with pericardial patch. In the mix type; pulmonary veins were anastomosed to the appropriate location of the left atrium due to anatomy.

Late term follow-ups were evaluated on medical charts and data were recorded. All patients were evaluated by transthoracic echocardiography (TTE) on the seventh day, first month and sixth month postoperatively. Patients without pathology were followed up on annual TTE. Cardiopulmonary catheterization was performed aiming to measure pressure to who had a defined pathology in pulmonary venous flow and/or left atrium diameters. The pediatric cardiology and cardiovascular surgeon's council evaluated the appropriate treatment decision.

\section{Statistical Analysis}

All data were presented with mean, standard deviation, median and interval data. Statistical analysis was performed using the SPSS 20.0 program.

\section{Results}

The mean age of the patients was determined as $9 \pm 11.68$ months. The mean weights of the patients before the operation were found to be $3720 \pm 2042$ gr. 24 of the patients were female and 17 were male.

Nineteen of the patients were supracardiac, 13 were intracardiac, 8 were infracardiac, and one was mixed type TAPVC. The demographic data of the patients are presented in Table 1.

Three patients underwent urgent surgical intervention for the first 24 hours after birth due to hemodynamic instability.

Definite diagnoses of the patients were detected during the operation. The diagnoses of the patients are given in Table 1. All patients were performed with total correction procedure for TAPVC pathology.

ASD repair was performed in all patients after pulmonary venous correction. In addition to these procedures, one patient had a ventricular septal defect
Table 1. Patient characteristics

\begin{tabular}{l|l}
\hline Caracteristics & Value \\
\hline $\begin{array}{l}\text { Sex (Male/Female) } \\
\text { Age (Months) }\end{array}$ & $17 / 24$ \\
\hline Wight (gr) & $9 \pm 11.68$ \\
& $3720 \pm 2042$ \\
\hline $\begin{array}{l}\text { Pulmonary Vein Anatomy } \\
\text { Supracardiac }\end{array}$ & $19(46 \%)$ \\
\hline $\begin{array}{l}\text { Intracardiac } \\
\text { İnfracardiac }\end{array}$ & $13(31 \%)$ \\
Mix & $8(19.5 \%)$ \\
& $1(3.5 \%)$ \\
\hline $\begin{array}{l}\text { Operative Details } \\
\text { Emergency surgery }\end{array}$ & \\
Concomittant surgery & $3(7 \%)$ \\
Cardiopulmonary bypass time & $38(93 \%)$ \\
Cross-clamp time & $84.78 \pm 25.84$ \\
\hline
\end{tabular}

Data are presented as mean $\pm S D$ or number

closure, emergent patent ductus arteriosus (PDA) division applied to three patients and bilateral bidirectional Glenn shunt procedure was applied to three patients.

All patients who underwent bidirectional Glenn shunt operation had heterotaxy syndrome and unbalanced ventricle.

The right pulmonary veins connected to the vena cava superior while the left pulmonary veins connected to the vena cava inferior together with the pulmonary sac in a patient with mixed TAPVC diagnosis. After three years of follow-up, expansion of the right pulmonary vein was performed due to stenosis in the anastomotic region. Postoperative follow-up for 3 years is not detected in anastomotic stricture and is followed by NYHA class I.

Mean cardiopulmonary by-pass time was $84.78 \pm 25.84$ minutes and mean aortic cross-clamp time was $57.24 \pm 15.17$ minute. Average intubation and intensive care unit stay times were measured as $15.9 \pm 34.28,52.97 \pm 33.49$ hours respectively, and hospital stay was detected $7.26 \pm 1.59$ in days (Table 2). During the hospital stay and during followup, two patients died of right ventricular failure and one patient died of malignant arrhythmia.

Five patients were admitted to the intensive care unit as an open sternum due to the fact that the heart was edematous and cause of low blood pressure when the 
Table 2. Operative and postoperative outcomes

\begin{tabular}{l|l} 
& $\mathbf{n}$ \\
\hline $\begin{array}{l}\text { Duration of ventilation (hours) } \\
\text { Duration in intensive care unit (hours) } \\
\text { Hospital stay (days) }\end{array}$ & $15.9 \pm 34.28$ \\
& $52.97 \pm 33.49$ \\
Complications & $7.26 \pm 1.59$ \\
Hemorrhagea & $14(34.1 \%)$ \\
Arrhythmia & 2 \\
Phrenic palsy & 1 \\
Failure to extubation & 1 \\
Delayed sternal closure & 3 \\
Nitric oxide therapy & 4 \\
& 3 \\
Hospital mortality & \\
Malignant arrhythmia & $3(7.3 \%)$ \\
Right ventricle failure & 1 \\
\hline Data are presented as mean \pm SD or number & 2
\end{tabular}

sternum closure is tried. The sternum of all patients was closed at the end of 48 hours postoperatively. One patient developed perioperative left ventricular failure.

In case of failure to release from cardiopulmonary bypass device the patient was followed by intensive care with ECMO. Due to no need of ECMO support anymore, it was terminated at the sixtieth hour postoperatively.

Three patients who were followed up for preoperative grade 2 pulmonary hypertension began to undergo inhaled nitric oxide treatment due to the persistence of postoperative pulmonary hypertension and extubation difficulty. Following terminating nitric oxide therapy, two patients were extubated at the hour postoperative forty eight. A tracheostomy procedure was performed to a patient with the reason of extubation difficulty. Postoperative complications patients are presented in Table 2.

\section{Discussion}

Although, surgical treatment of TAPVC provides acceptable hospital mortality rate and satisfactory outcomes in long term, patients with single ventricle physiology, presence of high degree pulmonary venous obstruction and heterotaxy syndrome still remain a challenge. ${ }^{(8)}$ In current era, a continuous improvement is reported in both mortality and morbidity rates in contemporary articles.
In accordance with the recent articles which stated a mortality rate between $5-13 \%$, we had mortality in 3 patients $(7.3 \%){ }^{(8-11)}$ Consequences of great collaboration in congenital heart-team such as more accurate preoperative diagnosis, development of preoperative stabilization and control of pulmonary hypertension by the administration of pulmonary vasodilators in the pre- and postoperative therapy, advanced operative techniques, and earlier operative approach contribute to achieve distinctly better results. ${ }^{(10)}$

Within this study, thereby presenting our single center outcomes of TAPVC following surgical correction, sought to determine possible risk factors causing late term complications and reoperation. Our study includes 41 patients with the diagnosis of TAPVC and has no excluding criteria. Previously, various factors have been associated with increased mortality rate, especially weight at operation $(<3 \mathrm{~kg})$, use of deep hypothermic circulatory arrest (DHCA) and prolonged aortic cross clamp (ACC) clamp time ( $>60$ minutes). ${ }^{(9,10,12)}$

There are controversies about the usage of DHCA, though most writers accepts it as a risk factor while some others including Lincoln et al. and Kirshbom et al. support the opposite. ${ }^{(12,13)}$ Particularly, we tried to avoid DHCA utilization; for this aim, when necessary, a vent cannula was placed into pulmonary venous sac for to keep the surgical field bloodless and paid maximum attention to complete the repair below an hour. Nevertheless, our mean ACC time was around 60 minutes. On the other hand, despite there was no significant difference, both $\mathrm{CPB}$ and $\mathrm{ACC}$ times were higher in mortality observed patients. However, we are in opinion that avoiding these factors helped us to achieve an admissible mortality rate.

On the other hand, mix type TAPVC often related to poorer outcomes. ${ }^{(4)}$ Unfortunately, our serial includes negligible quantity of -only one- patient with this diagnosis, thus it is hard to deduce sufficient results in the absence of adequate number of patient. However, a patient who underwent reoperation due to the pulmonary venous stenosis had mix type TAPVC. Our case had a significant 
stenosis at the anastomosis site of right pulmonary veins, hence required reoperation following three years after initial surgical approach. Afterwards, there was no remarkable gradient measured in aforementioned patient during last three years.

Khan et al. conducted a multi institutional study containing 65 centers and overall 261 patients. $^{(15)}$ The purpose was to assess the surgical outcomes in patients with heterotaxy syndrome. At the end, they declared increased mortality among patients with functionally univentricular physiology and heterotaxy syndrome. Various authors have confirmed that associated heterotaxy syndrome are worsening the survival. ${ }^{(16-18)}$ Nonetheless, need of postoperative extracorporeal membrane oxygenation, reoperation necessity for pulmonary vein stenosis and duration at hospital values were similar between groups. Moreover, they also did not observed a difference in patients who underwent emergent surgery within first 48 hours of birth.

Five patients of us were in single ventricle physiology and all had heterotaxy syndrome. The surgical process was completed by applying the bidirectional cava-pulmonary shunt. Three of them required urgent repair and those had concomitant PDA, therefore division was utilized. There was either mortality or need of ECMO support in this subgroup which is consisted of patients with heterotaxy syndrome/single ventricle physiology. The fact that remains that despite our results showing no difference in patients with heterotaxy syndrome, a comment should be made considering the sample size. Furthermore, outcomes of 3 patients with urgent surgical repair have to be evaluated in the same manner.

Some novel techniques have been recommended to prevent neointimal hyperplasia occurrence localized at the anastomosis site. For instance, a suturless technique that causing somewhat less trauma to the pulmonary vein and minimizes distortion risk at the anastomosis site has been reported by numerous authors albeit long-term surgical outcomes are unclear yet. ${ }^{(19)}$ Considering the suspects on this issue we are not tent to disuse the conventional method in favor of suturless technique imminently.
Nakayama et al. have identified possible risk factors for mortality regarding their cohort involving total 207 patients. ${ }^{20}$ They determined that body weight under 2.5 kgs was related to increase mortality. However all of our patients were weighing over that cut off value. Darling classification was performed in this study likewise we did. They determined that the type of TAPVC mostly class III and IV influence the early surgical outcome. As we mentioned before, revealed relation between mix type (IV) and improved mortality, contrary to usual knowledge, our cases that observed mortality were separately divided into class I, II and III for each.

Study of Karamlou et al. has to be a feature of unique entity thereby they have evaluated the effects of specific drugs in the immediate postoperative period in addition to mostly examined demographic data and operative factors. (9) Those were milrinone, epinephrine, and nitroprusside. Moreover, low cardiac output was defined as the main cause of early postoperative death, as in prior articles which highlighting the underlying rationale for increased use of inotropic agents. Due to the right cardiac failure in two cases and one with malign arrhythmia, we had total three early mortalities. However, we had to administer three patients nitric oxide therapy due to pulmonary hypertension and failure to extubation. Subsequently, two were able to extubate while the rest one needed a tracheostomy for prolonged intubation time.

This study includes some limitations due to a character of retrospective design. Moreover, our patient characteristics and associated anomalies were various and did not show equal distribution, therefore a healthy statistical analyze could not be performed. For these reasons, further prospective, controlled investigations should be performed involving large group of patients on this issue.

To sum up, the literature seems to be failed to build up a consensus about to determine definite predictive factors for mortality and morbidity in patients with TAPVC. Even, in large cohorts from multi center findings concerning long term results may be confusing. Developing in surgical 
era, improved perioperative care and collaboration of heart team have been improving event free survival day by day. When compared our results with the literature, we suggest that regardless of coexisting cardiac defects, surgical approach to all types of TAPVC at every age may be utilized safely and provides satisfactory results.

Declaration of Conflicting Interests: The authors declared no conflicts of interest with respect to the authorship and/or publication of this article.

Funding: The authors received no financial support for the research and/or authorship of this article.

\section{References}

1. Kelle AM, Backer CL, Gossett JG, Kaushal S, Mavroudis C. Total anomalous pulmonary venous connection: Results of surgical repair of 100 patients at a single institution. J Thorac Cardiovasc Surg 2010;139:138794.

2. Stein P. Total anomalous pulmonary venous connection. AORN J 2007;85:509-20.

3. Muller WH Jr. The surgical treatment of transposition of the pulmonary veins. Ann Surg 1951;134:683-93.

4. Padalino MA, Cavalli G, De Franceschi M, et al. Surgical Outcomes of Total Anomalous Pulmonary Venous Connection Repair: A 22 $\square$ Year Experience. J Card Surg 2014;29:678-85

5. Yoshimura N, Fukahara K, Yamashita A, et al. Current topics in surgery for isolated total anomalous pulmonary venous connection. Surg Today 2014;44:2221-6.

6. Shi G, Zhu Z, Chen J, et al. otal Anomalous Pulmonary Venous Connection: The Current Management Strategies in a Pediatric Cohort of 768 Patients. Circulation 2017;135:48-58.

7. Correa-Villasenor A, Ferencz C, Boughman JA, Neill CA. Total anomalous pulmonary venous return: familial and environmental factors. The Baltimore Washington Infant Study Group. Teratology 1991;44:415-28.
8. St Louis JD, Harvey BA, Menk JS, et al. Repair of "simple" total anomalous pulmonary venous connection: A review from the Pediatric Cardiac Care Consortium. Ann Thorac Surg 2012;94:133-7.

9. Karamlou T, Gurofsky R, Al Sukhni E, et al. Factors associated with mortality and reoperation in 377 children with total anomalous pulmonary venous connection. Circulation 2007;115:1591-8.

10. Seale AN, Uemura $\mathrm{H}$, Webber SA, et al. Total anomalous pulmonary venous connection: Morphology and outcome from an international populationbased study. Circulation 2010;21:2718-26.

11. Ades AM, Dominguez TE, Nicolson SC, et al. Morbidity and mortality after surgery for congenital cardiac disease in the infant born with low weight. Cardiol Young 2010;20:8-17.

12. Hoashi T, Kagisaki K, Oda T, et al. Long-term results of treatments for functional single ventricle associated with extracardiac type total anomalous pulmonary venous connection. Eur J Cardiothorac Surg 2013;43:965-70.

13. Lincoln CR, Rigby ML, Mercanti $\mathrm{C}$, et al. Surgical risk factors in total anomalous pulmonary venous connection. Am J Cardiol 1988;61:608-11

14. Kirshbom PM, Myung RJ, Gaynor JW, et al. Preoperative pulmonary venous obstruction affects long-term outcome for survivors of total anomalous pulmonary venous connection repair. Ann Thorac Surg 2002;74:1616-20.

15. Khan MS, Bryant R 3rd, Kim S, et al. Contemporary outcomes of surgical repair of total anomalous pulmonary venous connection in patients with heterotaxy syndrome. Ann Thorac Surg 2015;99:2134-40.

16. Lodge AJ, Rychik J, Nicolson SC, Ittenbach RF, Spray TL, Gaynor JW. Improving outcomes in functional single ventricle and total anomalous pulmonary venous connection. Ann Thorac Surg 2004;78:1688-95.

17. Morales DL, Braud BE, Booth JH, et al. Heterotaxy patients with total anomalous pulmonary venous return: improving surgical results. Ann Thorac Surg 2006;82:1621-7.

18. Jacobs JP, Pasquali SK, Morales DL, et al. Heterotaxy: lessons learned about patterns of practice and outcomes from the Congenital Heart Surgery Database of The Society of Thoracic Surgeons. World J Pediatr Congenit Heart Surg 2011;2:278-86

19. Yanagawa B, Alghamdi AA, Dragulescu A, et al. Primary sutureless repair for "simple" total anomalous pulmonary venous connection: midterm results in a single institution. J Thorac Cardiovasc Surg 2011;141:1346-54.

20. Nakayama Y, Hiramatsu T, Iwata Y, et al. Surgical results for functional univentricular heart with total anomalous pulmonary venous connection over a 25-year experience. Ann Thorac Surg 2012;93:606-13. 